\title{
Oblique route to turbulence
}

\author{
MUJEEB R. MALIK
}

NASA Langley Research Center, Hampton, VA 23681, USA

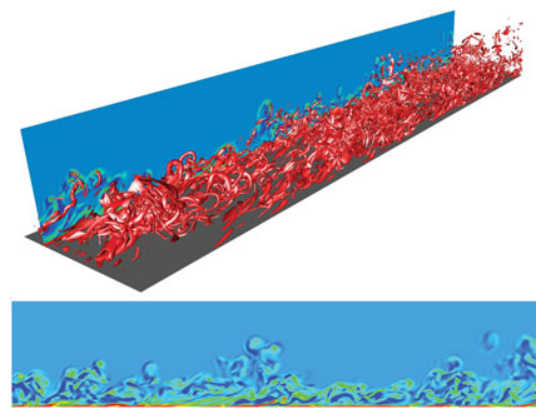

Direct numerical simulations have been performed by Mayer, Von Terzi \& Fasel (J. Fluid Mech., this issue, vol. 674, 2011, pp. 5-42) to demonstrate that oblique-mode breakdown leads to fully turbulent flow for a Mach 3 flat-plate boundary layer. Since very low level of initial disturbances is required for this transition scenario, obliquemode breakdown is the most potent mechanism for transition in two-dimensional supersonic boundary layers in low-disturbance environments relevant to flight.

Key words: boundary layer, linear stability, oblique waves, supersonic, transition

\section{Introduction}

Boundary-layer transition is particularly important in supersonic flows as it impacts not only vehicle drag but also skin temperatures requiring special attention to materials selection for high-Mach-number vehicles. Transition to turbulence is a complex process involving several stages: (i) internalization of external disturbances (e.g. acoustics, turbulence and wall roughness) in the boundary layer, which is known as 'receptivity'; (ii) growth of the internalized small-amplitude disturbances according to linear stability theory (LST); (iii) nonlinear evolution of boundarylayer disturbances followed by secondary instabilities and eventual breakdown to turbulence. Transition to turbulence in subsonic two-dimensional (2D) boundary layers, where Tollmien-Schlichting (TS) waves constitute the primary instability mechanism, is well understood. Two-dimensional TS waves are most amplified in a 2D subsonic boundary layer and, in this case, laminar-turbulent transition route involves amplification of TS waves to relatively large amplitude followed by secondary instabilities associated with fundamental or subharmonic resonances (Herbert 1988). The relevant mathematical model constitutes a 2D primary wave of large amplitude which is in resonance with two oblique waves with frequency equal to the primary wave frequency (fundamental secondary instability) or $1 / 2$ the primary wave frequency (subharmonic secondary instability). These secondary instabilities represent the first stage in the breakdown of laminar flow.

Mack (1969) investigated linear stability of supersonic boundary layers and showed that most amplified disturbances have large oblique angles with respect to the flow direction. Therefore, oblique modes amplify much more than 2D modes when Mach number is greater than unity. What will then be the most potent transition mechanism in such flows? The answer to this question was first attempted by a direct solution of Navier-Stokes equations for a Mach 1.6 boundary layer by Fasel, Thumm \& 
Bestek (1993). They showed that a pair of oblique modes can interact nonlinearly and lead to transition. Subsequently, this oblique-mode breakdown mechanism was studied by Chang \& Malik (1994) using parabolized stability equations. They showed that the primary breakdown mechanism in this case constitutes a wave-vortex triad consisting of two oblique modes and a steady streamwise vortex. The oblique waves of equal frequencies are linearly amplified while the vortex mode is generated by their nonlinear interaction. Higher harmonics are also generated by nonlinear interaction, which grow rapidly to reach amplitudes of the order of the amplitude of primary oblique waves. Laminar boundary layer begins to breakdown at this stage. Since oblique waves linearly amplify by several orders of magnitude in the boundary layer, the oblique-mode transition scenario can be initiated by disturbance amplitudes smaller than $0.01 \%$. In contrast, fundamental or subharmonic secondary instability mechanisms would require much higher initial amplitudes of the $2 \mathrm{D}$ mode in a supersonic boundary layer.

While in numerical simulations the oblique-mode breakdown appears to be most relevant for supersonic boundary layers, no directly controlled laboratory experiments (i.e. initiating the transition process with pairs of oblique waves) have been conducted to investigate this mechanism and therefore it is not known whether this mechanism can actually be realized. On the contrary, Kosinov et al. (1994) suggested the existence of an 'asymmetric subharmonic resonance' in their supersonic wind-tunnel experiment. Earlier direct numerical simulations (DNS) of supersonic boundary-layer transition have either concentrated on the linear and early nonlinear regime or the fully developed turbulent flows because it is difficult to simulate the entire transition regime due to large computational demands. However, such a simulation is necessary to answer the question whether the oblique-mode breakdown leads to a state that we recognize as a fully turbulent boundary layer. This has been the main contribution of Mayer et al. (2011). They have performed well-resolved numerical simulations employing up to 324 million grid points to show that oblique mode breakdown indeed leads to laminar-turbulent transition that results in a turbulent boundary layer, whose characteristics are consistent with general expectations and results from other direct numerical simulations.

\section{Overview}

Compressible Navier-Stokes equations were solved by using high-order methods to simulate boundary-layer transition accurately. For the direct numerical simulation, Mayer et al. (2011) chose a Mach number of 3 and a unit Reynolds number of $2.181 \times$ $10^{6} \mathrm{~m}^{-1}$ to match the physical conditions of an experiment conducted in the Princeton wind tunnel (Graziosi \& Brown 2002). The inflow of the computational domain was located downstream of the plate leading edge and thus the stage of receptivity to freestream disturbances was intentionally avoided. Instead, time-harmonic disturbances were introduced through a suction and blowing slot located on the wall downstream of the inflow plane. A pair of oblique waves with equal but opposite angles was forced through this slot and the frequency and wave angle of the forced disturbances were selected in order to allow strong amplification according to LST within the computational domain. Several cases were computed to study parametric effects and these cases employed hundreds of millions of grid points with a maximum of 324 million points to assure that small-scale structures that emerge during the transition process are captured accurately. In addition, high-order methods with very low 


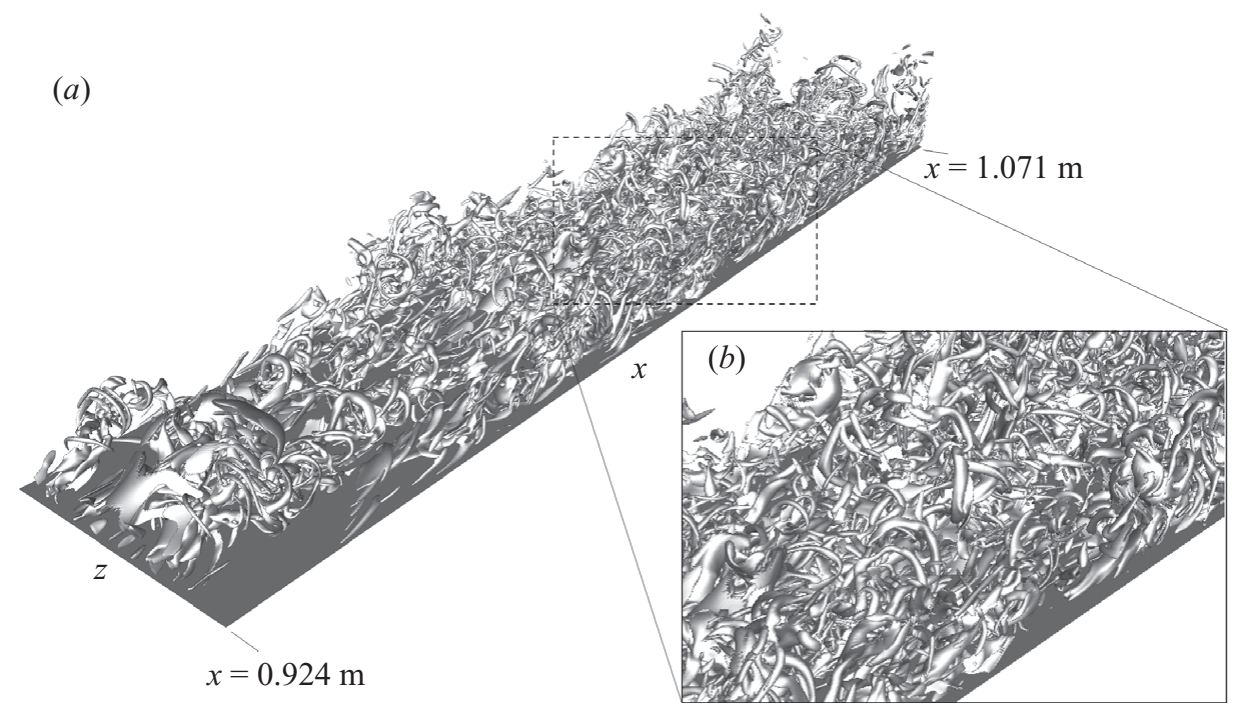

FIGURE 1. Computed instantaneous flow structures in the transitional and turbulent regimes: (a) entire three-dimensional view, $(b)$ close-up of the early turbulent region.

truncation error were used to minimize unwanted numerical disturbance forcing into the transition simulation.

In the DNS by Mayer et al. (2011), oblique-mode breakdown has been studied in meticulous detail, including initiation as oblique instability waves, wave-vortex interaction, generation of higher harmonics and eventual breakdown to turbulence. The computed turbulent state compares well with results in the open literature. For example, they found that the expected turbulent value of skin friction is achieved towards the end of the computation. Various aspects of the instantaneous flow structures in the transitional and turbulent regime are shown in the figure by the title, and in figure 1. The breakup is accompanied by a rapid increase in smallscale structures as illustrated in the figures. The late nonlinear stage in the spatially developing boundary layer is captured in great detail in the simulation. Computational results show that the 'breakup' of temporal periodicity occurs only downstream of the maximum skin friction and that the spectrum fills up rapidly at that stage with modes that are not direct descendants of the forced oblique waves and the subsequent nonlinear interactions. What is important here is that the oblique breakdown does lead to turbulent flow, which demonstrates that the oblique breakdown mechanism is a viable and relevant transition mechanism in 2D supersonic boundary layers in low free-stream disturbance environments (e.g. free flight).

With reference to figure 1, transition begins (i.e. a rise in skin-friction) at $x=0.74 \mathrm{~m}$ corresponding to a Reynolds number of $1.6 \times 10^{6}$, which is much less than the value of $12 \times 10^{6}$ reported by Chen, Malik \& Beckwith (1989) for the Mach 3.5 quiet tunnel experiment. It is clear that higher forcing amplitudes were used by Mayer et al. to capture the transition mechanism of interest in a relatively small computational domain. A DNS of the entire transition process (covering receptivity, linear/nonlinear growth and turbulence) with forcing amplitudes relevant to flight would have required a much longer computational domain and billions of grid points. Such a simulation should soon be possible as additional computational resources become available. 


\section{Future}

With the ever increasing computational power, DNS is fast becoming a powerful tool for investigation of complex phenomena involving transitional and turbulent flows. The work of Mayer et al. (2011) is an impressive application of DNS where the complete transition process is simulated and it is demonstrated that the oblique route to turbulence is a relevant mechanism for supersonic boundary-layer transition. The computed turbulent state in this case of Mach 3 is expected to behave as incompressible turbulence based on Morkovin's hypothesis (see Fernholz \& Finley 1980) and the computational results do support this conjecture since the maximum fluctuation Mach number reaches a value of only about 0.3. However, Morkovin's hypothesis is expected to be violated at high Mach numbers and DNS could prove valuable in understanding turbulence physics in hypersonic boundary layers and provide useful data for modelling of such flows. In hypersonic boundary layers, 2D acoustic modes are most amplified and, therefore, secondary instabilities may play a dominant role in the transition process.

In the area of boundary-layer transition prediction, what is needed is a complete simulation covering the entire transition regime (from free-stream disturbance forcing to fully developed turbulent flow). Such a simulation, coupled with the knowledge of external disturbance environment, would provide true prediction of transition. However, prescription of the free-stream environment is an unsolved problem and, therefore, transition prediction will remain to be a challenge for some time to come.

\section{References}

Chang, C. L. \& Malik, M. R. 1994 Oblique-mode breakdown and secondary instability in supersonic boundary layers. J. Fluid Mech. 273, 323-360.

Chen, F. J., Malik, M. R. \& Beckwith, I. E. 1989 Boundary layer transition on a cone and flat plate at Mach 3.5. AIAA J. 27(6), 687-693.

Fasel, H., Thumm, A. \& Bestek, H. 1993 Direct numerical simulation of transition in supersonic boundary layer: oblique breakdown. In Transitional and Turbulent Compressible Flows (ed. L. D. Kral \& T. A. Zang), FED, vol. 151, pp. 77-92, ASME.

Fernholz, H. H. \& Finley, P. J. 1980 A critical commentary on mean flow data for two-dimensional compressible turbulent boundary layers. AGARD Rep. 254. Advisory Group for Aerospace Research and Development.

Graziosi, P. \& Brown, G. L. 2002 Experiments on stability and transition at mach 3. J. Fluid Mech. 472, 83-124.

Herbert, T. 1988 Secondary instability of boundary layers. Annu. Rev. Fluid Mech. 20, 487-526.

Kosinov, A. D., Seminov, N. V., Shevelkov, S. G. \& Zinn, O. I. 1994 Experiments on the nonlinear instability of supersonic boundary layers. In Nonlinear Instability of Nonparallel Flows (ed. D. T. Valentine, S. P. Lin \& W. R. C. Philips), pp. 196-205. Springer.

Mack, L. M. 1969 Boundary-layer stability theory. In Internal Document 900-277. Jet Propulsion Laboratory.

MaYer, C. S. J., Von Terzi, D. A. \& Fasel, H. F. 2011 DNS of complete transition to turbulence via oblique breakdown at Mach 3. J. Fluid Mech. 674, 5-42. 